\title{
EW and mixed QCD-EW effects in the W boson mass determination
}

Mauro Chiesa*†

Julius-Maximilians-Universität Würzburg, Institut für Theoretische Physik und Astrophysik, D-97074 Würzburg, Germany.

E-mail: mauro.chiesa@physik.uni-wuerzburg.de

Carlo Michel Carloni Calame, Fulvio Piccinini, Oreste Nicrosini

INFN, Sezione di Pavia, Via A. Bassi 6, 27100, Pavia, Italy

E-mail: carlo.carloni.calameepv.infn.it,fulvio.piccininiepv.infn.it,

oreste.nicrosiniepv.infn.it

\section{Homero Martinez, Guido Montagna}

Dipartimento di Fisica, Università di Pavia, and INFN, Sezione di Pavia, Via A. Bassi 6, 27100,

Pavia, Italy

E-mail: guido.montagna@pv.infn.it

\section{Alessandro Vicini}

Tif lab, Dipartimento di Fisica, Università di Milano, and INFN, Sezione di Milano, Via G.

Celoria 16, 20133, Milano, Italy

E-mail: alessandro.vicini@mi.infn.it

We present some selected results from Ref. [1] where we performed a comprehensive analysis of the impact of the QED, electroweak and mixed QCD-electroweak corrections to the determination of the $\mathrm{W}$ boson mass at hadron colliders.

Sixth Annual Conference on Large Hadron Collider Physics (LHCP2018)

4-9 June 2018

Bologna, Italy

* Speaker.

${ }^{\dagger}$ M. C. would like to thank the organizers for their kind invitation. 


\section{Introduction}

The $\mathrm{W}$ boson mass $\left(M_{\mathrm{W}}\right)$ has been measured by the CDF [2] and D $\varnothing$ [3] experiments at TEVATRON with an experimental error of $19 \mathrm{Mev}$ and $23 \mathrm{MeV}$, respectively. The ATLAS collaboration at CERN published a measurement of $M_{\mathrm{W}}$ with an error of $19 \mathrm{MeV}$ [4] and both the ATLAS and the CMS experiments are planning to measure $M_{\mathrm{W}}$ with a final error of $15 \mathrm{MeV}$ (or eventually $10 \mathrm{MeV}$ ). Such a precision requires a careful assessment of the theoretical uncertainties that affect the $\mathrm{W}$ mass determination.

At hadron colliders $M_{\mathrm{W}}$ is measured from distributions like the charged lepton $p_{\mathrm{T}}$ or the leptonneutrino transverse mass $\left(M_{\mathrm{T}}\right)$ in charged Drell-Yan production using a template fit procedure. The templates are generated by Monte Carlo event generators with a given theoretical accuracy which are affected by theoretical uncertainties. On the one hand, there are perturbative uncertainties coming from the missing higher-order corrections and, on the other hand, there are also non-perturbative uncertainties such as the ones related to the PDFs or the modeling of the $p_{\mathrm{T}}$ of the $\mathrm{W}$ boson. These uncertainties propagate to the $\mathrm{W}$ mass measurement.

\section{Technical details of the calculation and tools}

Our strategy for the determination of the theoretical uncertainties in the $\mathrm{W}$ mass measurement follows the experimental procedure. We consider two sets of Monte Carlo samples: the first one corresponds to the pseudodata and play the rôle of data in our analysis, while the samples in the other set are the templates. The templates are generated with different values of $M_{\mathrm{W}}$ using a reweighting procedure ${ }^{1}$ and are fitted to the pseudodata. The theoretical uncertainties are defined as the shifts in the $\mathrm{W}$ mass $\left(\Delta M_{\mathrm{W}}\right)$ that are the difference between the nominal value of $M_{\mathrm{W}}$ in the pseudodata and the result of the fit. We focus on the perturbative uncertainties of electroweak (EW) and mixed QCD-EW origin. The event samples are produced with the event generators HORACE [5, 6] and POWHEG-BOX-V2 [7, 8, 9] (W_EW-BMNNP package [10]).

HORACE is a Monte Carlo event generator for Drell-Yan production that can generate events at NLO EW accuracy matched with QED Parton Shower (PS). The fully differential HORACE result can be cast in the form:

$$
d \sigma_{\mathrm{HORACE}}=d \sigma_{0}\left[1+\delta_{\alpha}+\sum_{n=2}^{\infty} \delta_{\alpha^{n}}^{\prime}\right]
$$

where $\delta_{\alpha}$ stands for the one-loop EW corrections, while the sum in Eq. (2.1) represents the QED PS contribution containing soft and virtual multiple-photon radiation in leading logarithmic approximation. Note that the matching procedure replaces the first PS radiation with the exact $\mathscr{O}(\alpha)$ one.

The W_EW-BMNNP package of POWHEG-BOX-V2 is an event generator for charged Drell-Yan that can produce events at NLO QCD+NLO EW accuracy matched with both QCD and QED PS. The POWHEG predictions can be written as follows:

$$
d \sigma_{\mathrm{POWHEG}}=d \sigma_{0}\left[1+\delta_{\alpha_{s}}+\delta_{\alpha}+\sum_{m=1, n=1}^{\infty} \delta_{\alpha_{s}^{m} \alpha^{n}}^{\prime}+\sum_{m=2}^{\infty} \delta_{\alpha_{s}^{m}}^{\prime}+\sum_{n=2}^{\infty} \delta_{\alpha^{n}}^{\prime}\right] .
$$

\footnotetext{
${ }^{1}$ The reweighting is well defined since the templates never include EW corrections.
} 


\begin{tabular}{rclr}
\hline & Templates & Pseudodata & $M_{W}$ shifts (MeV) \\
\hline 1 & LO & POWHEG(QCD) NLO & $56.0 \pm 1.0$ \\
2 & LO & POWHEG(QCD)+PYTHIA(QCD) & $74.4 \pm 2.0$ \\
3 & LO & HORACE(EW) NLO & $-94.0 \pm 1.0$ \\
4 & LO & HORACE (EW,QEDPS) & $-88.0 \pm 1.0$ \\
5 & LO & POWHEG(QCD,EW) NLO & $-14.0 \pm 1.0$ \\
6 & LO & POWHEG(QCD,EW) two-rad+PYTHIA(QCD)+PHOTOS & $-5.6 \pm 1.0$ \\
\hline
\end{tabular}

Table 1: $\mathrm{W}$ mass shifts (in $\mathrm{MeV}$ ) induced by different sets of perturbative corrections and evaluated with templates computed at LO. $M_{\mathrm{W}}$ is extracted from the $M_{\mathrm{T}}$ distribution in $\mu^{+} v$ production at the LHC at $14 \mathrm{TeV}$.

Besides the NLO EW+QED PS and the NLO QCD+QCD PS corrections, Eq. (2.2) contains a mixed QCD-EW contribution coming from the application of a QED (QCD) PS on events including NLO QCD (NLO EW) corrections.

While HORACE provides an internal implementation of the QED PS, POWHEG relies on external shower Monte Carlo programs, that in our calculation are PYTHIA8 [11] and PYTHIA8 in combination with PHOTOS $[12,13]$.

\section{Mixed QCD-EW corrections}

Table 1 summarizes the shifts in the $\mathrm{W}$ mass extracted from the $M_{\mathrm{T}}$ distribution in the bare muon channel corresponding to several classes of higher-order corrections ranging from the fixedorder NLO QCD and/or NLO EW to the full simulation at NLO QCD+NLO EW matched with QCD and QED PS. From Tab. 1 it is possible to estimate the impact of the mixed QCD-EW corrections in Eq. (2.2). We find that the mixed QCD-EW contributions introduce a shift of $-16 \pm 3 \mathrm{MeV}$, in agreement with the results of Ref. [14] (-14 MeV) where the two-loop corrections $\mathscr{O}\left(\alpha \alpha_{\mathrm{S}}\right)$ were computed in pole approximation.

\section{Results for the LHC}

In Table $2 M_{\mathrm{W}}$ is extracted from the charged lepton $p_{\mathrm{T}}$ and from the $M_{\mathrm{T}}$ distributions in the bare muon and in the dressed electron setup. The templates are generated at NLO QCD+QCD PS, while the pseudodata are generated at NLO QCD+QCD PS+QED PS (samples [1]-[2]) and at NLO QCD+NLO EW matched with QCD and QED PS (samples [3]-[4]).

The differences between lines [1] and [2] ([3] and [4]) in Tab. 2 come from the different implementation of the QED PS in PYTHIA and in PHOTOS. The numerical impact of these differences on the $\mathrm{W}$ mass determination is large in the setup of lines [1]-[2], while it is strongly reduced in the setup of lines [3]-[4], where the first radiation is provided by POWHEG at NLO EW and the two predictions start to differ at $\mathscr{O}\left(\alpha^{2}\right)$.

Taking the difference between lines [3] and [1] (or [4] and [2]) we get an estimate of the impact of the non-logarithmic QED corrections, weak corrections and mixed QCD-EW corrections. The numerical results are summarized in Tab. 3. 


\begin{tabular}{|c|c|c|c|c|c|c|}
\hline \multirow{2}{*}{\multicolumn{3}{|c|}{$\begin{array}{c}\qquad p p \rightarrow W^{+}, \sqrt{s}=14 \mathrm{TeV} \\
\text { Templates accuracy: NLO-QCD+QCDPS }\end{array}$}} & \multicolumn{4}{|c|}{$M_{\mathrm{W}}$ shifts $(\mathrm{MeV})$} \\
\hline & & & \multicolumn{2}{|c|}{$W^{+} \rightarrow \mu^{+} v$} & \multicolumn{2}{|c|}{$W^{+} \rightarrow e^{+} v($ dres $)$} \\
\hline & Pseudodata accuracy & QED FSR & $M_{T}$ & $p_{T}^{l}$ & $M_{T}$ & $p_{T}^{l}$ \\
\hline 1 & $\mathrm{NLO}-\mathrm{QCD}+(\mathrm{QCD}+\mathrm{QED})_{\mathrm{PS}}$ & PYTHIA & $-95.2 \pm 0.6$ & $-400 \pm 3$ & $-38.0 \pm 0.6$ & $-149 \pm 2$ \\
\hline 2 & $\mathrm{NLO}-\mathrm{QCD}+(\mathrm{QCD}+\mathrm{QED})_{\mathrm{PS}}$ & PHOTOS & $-88.0 \pm 0.6$ & $-368 \pm 2$ & $-38.4 \pm 0.6$ & $-150 \pm 3$ \\
\hline 3 & NLO-(QCD+EW)+(QCD+QED) $)_{P S}$ two-rad & PYTHIA & $-89.0 \pm 0.6$ & $-371 \pm 3$ & $-38.8 \pm 0.6$ & $-157 \pm 3$ \\
\hline 4 & NLO-(QCD+EW)+(QCD+QED) $)_{P S}$ two-rad & PHOTOS & $-88.6 \pm 0.6$ & $-370 \pm 3$ & $-39.2 \pm 0.6$ & $-159 \pm 2$ \\
\hline
\end{tabular}

Table 2: $\mathrm{W}$ mass shifts (in MeV) induced by multiple QED FSR and mixed QCD-EW corrections at the $\mathrm{LHC}$ at $14 \mathrm{TeV}$.

\begin{tabular}{cccc}
\hline & & \multicolumn{2}{c}{$\Delta M_{\mathrm{W}}(\mathrm{MeV})$ bare muons } \\
\hline & QED FSR model & $M_{T}$ & $p_{T}^{l}$ \\
\hline \multirow{2}{*}{ LHC } & PYTHIA & $+6.2 \pm 0.8$ & $+29 \pm 4$ \\
& PHOTOS & $-0.6 \pm 0.8$ & $-2 \pm 4$ \\
\hline
\end{tabular}

Table 3: Impact of the non-logarithmic QED corrections, weak corrections and mixed QCD-EW corrections on the $\mathrm{W}$ mass determination at the $\mathrm{LHC}$ at $14 \mathrm{TeV}$ in the bare muon setup.

\section{Higher order EW corrections}

All possible consistent choices of input parameter scheme are equivalent at a given order in perturbation theory and the numerical differences between the predictions in these schemes are higher-order effects. In Ref. [1] we considered the $\alpha_{0}$ scheme (with $\alpha_{0}=1 / 137.035999074$ ) and two variants of the $G_{\mu}$ scheme, with $\alpha_{\mu}^{\text {tree }}=\sqrt{2} G_{\mu} M_{\mathrm{W}}^{2} \sin ^{2} \theta_{W} / \pi$ and $\alpha_{\mu}^{1-\text { loop }}=\alpha_{\mu}^{\text {tree }}(1-\Delta r)$, respectively. In all the three schemes, the coupling between final state photons and fermions is $\alpha_{0}$. The theoretical uncertainties on the $\mathrm{W}$ mass determination are collected in Tab. 4. The shifts in $M_{\mathrm{W}}$ are of order $10 \mathrm{MeV}$ at NLO EW, while they decrease down to $2 \mathrm{MeV}$ at NLO EW+QED PS.

The unresolved radiation of a lepton pair is an $\mathscr{O}\left(\alpha^{2}\right)$ effect that has been implemented in HORACE using a running value of $\alpha$ together with appropriate modifications of the Sudakov form factor. The shifts in $M_{\mathrm{W}}$ due to lepton-pair radiation are collected in Tab. 5, where we see an effect of approximately $5 \mathrm{MeV}$ in the muon setup and $3 \mathrm{MeV}$ in the electron setup.

\section{Conclusions}

We reported on the results of Ref. [1], where we studied the theoretical uncertainties on the W boson mass determination coming from QED, EW and mixed QCD-EW corrections. The Monte Carlo samples used at the LHC for the determination of $M_{\mathrm{W}}$ are generated at NLO QCD+QCD PS+QED PS: we found that the missing non-logarithmic QED corrections, weak corrections and mixed QCD-EW corrections introduce a theoretical uncertainty of a few $\mathrm{MeV}$ depending on the 


\begin{tabular}{ccc|cc}
\hline \multicolumn{3}{c|}{$p \bar{p} \rightarrow W^{+}, \sqrt{s}=1.96 \mathrm{TeV}$} & \multicolumn{2}{c}{$M_{\mathrm{W}}$ shifts $(\mathrm{MeV})$} \\
Templates accuracy: LO & \multicolumn{2}{c}{$W^{+} \rightarrow \mu^{+} v$} \\
& Pseudodata accuracy & Input scheme & $M_{T}$ & $p_{T}^{l}$ \\
\hline 1 & HORACE NLO-EW & $\alpha_{0}$ & $-101 \pm 1$ & $-117 \pm 2$ \\
2 & & $G_{\mu}-I$ & $-112 \pm 1$ & $-130 \pm 1$ \\
3 & & $G_{\mu}-I I$ & $-101 \pm 1$ & $-117 \pm 1$ \\
4 & HORACE NLO-EW+QED-PS & $\alpha_{0}$ & $-70 \pm 1$ & $-81 \pm 1$ \\
5 & & $G_{\mu}-I$ & $-72 \pm 2$ & $-83 \pm 1$ \\
6 & & $G_{\mu}-I I$ & $-72 \pm 1$ & $-82 \pm 2$ \\
\hline
\end{tabular}

Table 4: W mass shifts (in MeV) induced by different choices of the input parameter scheme at the TEVATRON.

\begin{tabular}{ll|cc|cc}
\hline \multirow{2}{*}{$p p \rightarrow W^{+}, \sqrt{s}=14 \mathrm{TeV}$} & \multicolumn{4}{c}{$M_{\mathrm{W}}$ shifts $(\mathrm{MeV})$} \\
\multicolumn{2}{c}{\begin{tabular}{c} 
Templates accuracy: LO \\
\multicolumn{2}{c}{ Pseudo-data accuracy }
\end{tabular}} & \multicolumn{2}{|c}{$W^{+} \rightarrow \mu^{+} v$} & \multicolumn{2}{c}{$W^{+} \rightarrow e^{+} v$} \\
\hline 1 & HORACE FSR-LL & $-89 \pm 1$ & $-97 \pm 1$ & $-179 \pm 1$ & $-195 \pm 1$ \\
2 & HORACE FSR-LL + Pairs & $-94 \pm 1$ & $-102 \pm 1$ & $-182 \pm 2$ & $-199 \pm 1$ \\
\hline
\end{tabular}

Table 5: W mass shifts (in MeV) induced by lepton-pair radiation at the $\mathrm{LHC}$ at $14 \mathrm{TeV}$.

setup and on the tools used for the description of multiple QED FSR. We found that our approximated estimate of the mixed QCD-EW corrections is in agreement with the results of Ref. [14] based on an NNLO calculation in pole approximation. We also studied the impact on the W boson mass determination of higher-order effects such as pair radiation.

\section{Acknowledgments}

The work presented in this talk was supported in part by the Italian Ministry of University and Research under the PRIN project 2010YJ2NYW. AV is supported by the European Commission through the HiggsTools Initial Training Network PITN-GA2012-316704. The work of HM has been supported by the Research Executive Agency (REA) of the European Union under the Grant Agreement number PITN-2010- 264564 (LHCPhenoNet) and by the University of Pavia under the grant "Fondo Giovani".

\section{References}

[1] C. M. Carloni Calame, M. Chiesa, H. Martinez, G. Montagna, O. Nicrosini, F. Piccinini and A. Vicini, Precision Measurement of the W-Boson Mass: Theoretical Contributions and Uncertainties, Phys. Rev. D 96 (2017) no.9, 093005 [arXiv:1612.02841 [hep-ph]].

[2] T. A. Aaltonen et al. [CDF Collaboration], Precise measurement of the $W$-boson mass with the Collider Detector at Fermilab, Phys. Rev. D 89 (2014) no.7, 072003 [arXiv:1311.0894 [hep-ex]].

[3] V. M. Abazov et al. [D0 Collaboration], Measurement of the W boson mass with the D0 detector, Phys. Rev. D 89 (2014) no.1, 012005 [arXiv:1310.8628 [hep-ex]]. 
[4] M. Aaboud et al. [ATLAS Collaboration], Measurement of the W-boson mass in pp collisions at $\sqrt{s}=7$ TeV with the ATLAS detector, Eur. Phys. J. C 78 (2018) no.2, 110 [arXiv:1701.07240 [hep-ex]].

[5] C. M. Carloni Calame, G. Montagna, O. Nicrosini and A. Vicini, Precision electroweak calculation of the charged current Drell-Yan process, JHEP 0612 (2006) 016 [hep-ph/0609170].

[6] C. M. Carloni Calame, G. Montagna, O. Nicrosini and A. Vicini, Precision electroweak calculation of the production of a high transverse-momentum lepton pair at hadron colliders, JHEP 0710 (2007) 109 [arXiv:0710.1722 [hep-ph]].

[7] P. Nason, A New method for combining NLO QCD with shower Monte Carlo algorithms, JHEP 0411 (2004) 040 [hep-ph/0409146].

[8] S. Frixione, P. Nason and C. Oleari, Matching NLO QCD computations with Parton Shower simulations: the POWHEG method, JHEP 0711 (2007) 070 [arXiv:0709.2092 [hep-ph]].

[9] S. Alioli, P. Nason, C. Oleari and E. Re, A general framework for implementing NLO calculations in shower Monte Carlo programs: the POWHEG BOX, JHEP 1006 (2010) 043 [arXiv:1002.2581 [hep-ph]].

[10] L. Barze, G. Montagna, P. Nason, O. Nicrosini and F. Piccinini, Implementation of electroweak corrections in the POWHEG BOX: single W production, JHEP 1204 (2012) 037 [arXiv:1202.0465 [hep-ph]].

[11] T. Sjostrand, S. Mrenna and P. Z. Skands, A Brief Introduction to PYTHIA 8.1, Comput. Phys. Commun. 178 (2008) 852 [arXiv:0710.3820 [hep-ph]].

[12] E. Barberio and Z. Was, PHOTOS: A Universal Monte Carlo for QED radiative corrections. Version 2.0, Comput. Phys. Commun. 79 (1994) 291.

[13] P. Golonka and Z. Was, PHOTOS Monte Carlo: A Precision tool for QED corrections in $Z$ and $W$ decays, Eur. Phys. J. C 45 (2006) 97 [hep-ph/0506026].

[14] S. Dittmaier, A. Huss and C. Schwinn, Dominant mixed QCD-electroweak $\mathscr{O}\left(\alpha \alpha_{\mathrm{S}}\right)$ corrections to Drell-Yan processes in the resonance region, Nucl. Phys. B 904 (2016) 216 [arXiv:1511.08016 [hep-ph]]. 